\title{
Experimental Evaluations of Touch Interaction Considering Automotive Requirements
}

\author{
Andreas Haslbeck, Severina Popova, Michael Krause, Katrina Pecot, \\ Jürgen Mayer, and Klaus Bengler \\ Institute of Ergonomics, Technische Universität München, \\ Boltzmannstrasse 15, 85747 Garching, Germany \\ \{Haslbeck, bengler\}@tum.de, \{popova, Krause\} alfe.mw.tum.de, \\ kjpecot@gmail.com, Juergen. Mayer@eu-bs.de
}

\begin{abstract}
Three different usability studies present evaluation methods for cross-domain human-computer-interaction. The first study compares different input devices like touch screen, turn-push-controller or handwriting recognition under regard of human error probability, input speed and subjective usability assessment. The other experiments had a focus on typical automotive issues: interruptibility and the influence of oscillations of the cockpit on the interaction.
\end{abstract}

Keywords: Touch, input medium, efficiency, effectiveness.

\section{Introduction}

The evaluation of different input devices is a classical question in usability experiments. Meanwhile a multiplicity of alternatives for most different employment scenarios exists. Nowadays such input devices are not only taken for human-computerinteraction (HCI) at PC workstations, but they are spread over many different professional applications, like cars, machinery, industrial workplaces and also taken for leisure and mobile applications. A recent example is Apple's ${ }^{\text {tm }}$ launch of the iPhone: touch-based interaction became popular and so it now emerges in more and more applications especially at mobile devices and in new domains. Above all, many new gadgets address user experience and an emotional channel of its potential users.

One focus on these trends is automotive driven: which input device is the best medium for driver's interaction with In-vehicle Information Systems (IVIS). Within this domain, different use-cases exist, beginning from cars to heavy trucks and construction machinery. All these scenarios have different requirements on usability.

This paper presents three experiments, which focus on effectiveness and efficiency of different input devices depending on the context where they are to be taken in use. The focus is on the one hand on typical operating steps towards IVIS and on the other hand on different environmental conditions which are typical for an automotive application. The different experimental settings are also recommendations for automotive HCI designers to evaluate future interfaces in early stages of product development. 


\section{Present Situation}

All devices and use-cases have in common that their usability strongly depends on the man-machine-interface (MMI). The most important mean of communication is a hand-based interaction via touch media or different turn-push-controllers. Past alternatives are mechanical hardware buttons, levers and rotating discs, while speech recognition has in fact been implemented, but is rather a future trend and has not yet reached full performance.

A previous study, by Kavakli and Thorne [1] compared the user's performance on computer video games using different input devices. It revealed that the kind of game has an influence on the best input device to take in order to improve user's performance. Nowadays another important focus lies on the age of users: the question is whether the user's age has an influence on operator's performance with different input devices. Murata and Iwase [2] showed that users of different age groups revealed no significant differences in performance at a pointing time experiment on touch panels, while they did on the same setting, operating with a computer mouse. In addition, they also stated that learning time required by a touch panel was smaller. In contrary to these findings, an experiment conducted by Armbrüster et al [3] found differences depending on the age while different tasks (click and drag \& drop) had to be done via touchpad. Rogers et al [4] concluded in studies similar to the following experiment, that younger users are able to show faster input times than older ones.

\section{Alphanumeric Input}

In a first experimental setting, different input media have been examined during alphanumeric inputs concerning effectiveness, efficiency and satisfaction in a singletask situation. The participants had to write down texts under the demand of a minimum of faults and a maximum of typed characters. So the subjects had to handle a speed-accuracy-tradeoff. Among the different input devices, a traditional keyboard, a touch screen keyboard on laptop and iPod Touch, a knob like it is equipped in cars and a handwriting recognition software on laptop were taken into account.

\subsection{Participants}

The test subjects have been university employees and students. 15 participants between the ages of 24 and 60 (mean $=31.7, \mathrm{SD}=11.1)$ took part in this study. There were a total of 12 males and 3 females. All the participants had normal or corrected to normal visual acuity and gave their consent for the experiment. Participation was done on a voluntary basis.

\subsection{Experimental Design and Methods}

The subjects were required to use five different devices for text input. These were a Lenovo S10-3T touch screen (TS) tablet PC featuring a 10 inch display with two text input options (touch-keyboard and handwriting recognition), a traditional Dell QWERTY keyboard, a knob-like device reminiscent of the BMW iDrive (see fig. 3) 
but with different mechanical qualities and in order to have comparison to a leisure gadget, an Apple iPod Touch (see fig. 4). With the knob device, the users scrolled through a wheel of letters and pressed down on the knob to select the intended letter. While handwriting, the user wrote by finger on the screen and the computer software recognized the writing and converted it to text. The hardware keyboard and the knob were attached to the laptop so all alphanumerical inputs could be done by the laptop with the exception of the iPod. While writing on the iPod the decision to hold the iPod upright or crosswise was left to the participants.

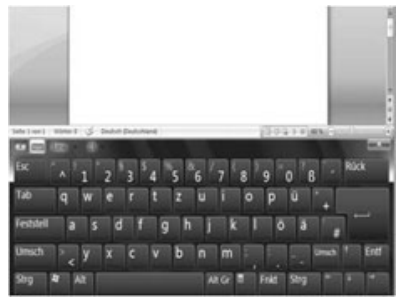

Fig. 1. Touch keyboard

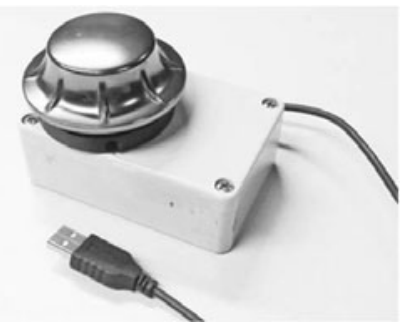

Fig. 3. Tentative turn-push-controller

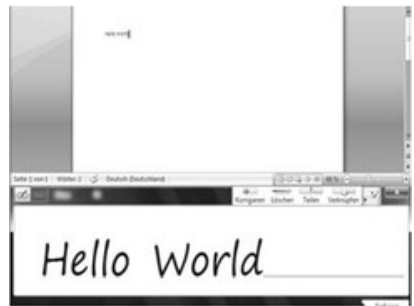

Fig. 2. Handwriting recognition form

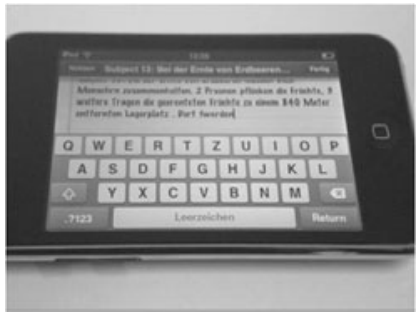

Fig. 4. iPod Touch

In the setup of the experimental apparatus, the tablet-PC was located in front of the participant at a comfortable viewing distance. It was placed on a wedge at a 45 degree angle to allow viewing the laptop in an angle of nearly 90 degrees. An additional LCD screen was present right in front of the subjects, which displayed the alphanumeric text that the user was asked to copy in each trial with the present device. In total, there were five different alpha-numeric input texts. The texts consisted of simple sentences from sources such as newspapers and books, containing several numbers, for example short news from a football match. The order of administration of the device and text sentences was randomized for each user.

With exception of the iPod, the text input progress was observed for each device by the test administrator on another LCD screen that duplicated the laptop screen.

The research question of this experiment is, whether the kind of input device has an influence on human error probability (HEP) and input speed. The subjects had three minutes to write on each device after the chance of getting familiarized with all 
devices. The instructor noted the exact word/number when passing the first and the second minute of each trial in order to have information about writing progress every minute. The assessment of the user's performance at the TS handwriting recognition has limits: a typing error, which is common for keyboards, does not exist there. The reason is that every input is processed by the software and afterwards interpreted as an expression into the set language. On account of this, here the counted errors are mistaken words by the resulting inputs after the recognition software process. So many counted errors have its origin in spidery handwriting, but less in problems of typing or spelling words or the design of this input device. The subjects were also told not to care for errors while typing in order to waste no time.

For subjective measurement of the experiment, participants completed the System Usability Survey (SUS) by Brooke [5]. The SUS is a simple, ten-item Likert scale which gives a global view of the subjective assessment of the usability of a device.

Objective measurement has been done by text analysis after the experiments. For each input medium, the average input speed was calculated in characters per minute. This was done by counting the total characters and spaces typed with each device per participant, then dividing the total characters by three and averaging them for each medium. Looking at table 1 , it can be seen that the keyboard had the fastest input. The TS keyboard, TS handwriting recognition and iPod had relatively similar input speeds, and the knob had the slowest input speed, which, in comparison to the keyboard, only had about $10 \%$ of whose input speed.

The HEP, which is defined as the ratio between the number of actual occurred errors and the number of given opportunities for errors to occur, was calculated for each input device. To obtain this number, the total errors and characters were each summed across the participants on each input medium. Then they were divided (total errors across participants/total characters performed on the medium across participants) to obtain the HEP. In table 1 can be seen that the keyboard had the lowest error probability. The iPod had the second lowest HEP. The remaining input mediums, TS handwriting, TS keyboard and knob, had the highest HEPs.

Finally a correlation analysis was performed on the data for each input medium to determine if there was a correlation between the speed of input and errors performed. From these analyses, two input devices (the hardware keyboard and TS keyboard) showed strong positive correlations of $\mathrm{r}_{\mathrm{p}}=.702$ with $\mathrm{p}=.004$ (hardware keyboard) and $r_{p}=.731$ with $p=.002$ (TS keyboard). Therefore, as the speed of input increased, the number of errors increased. The other devices showed no significantly strong correlation values.

These results show the advantages of a hardware keyboard in comparison to all other input devices: it enables a high input speed combined with low error rates. So this is the strong recommendation for workplaces where this type of keyboard is applicable. In spite of these facts, the user's choice can be different: in leisure application the mentioned performance measures don't count. Here, look and hedonic aspects are crucial. An additional AttrakDiff [6] evaluation of the different input devices has shown, that in terms of hedonic quality, the handwriting recognition, the iPod Touch and the TS keyboard have gained very good results, much better than the hardware keyboard. And finally, these results are valid only for single-task situations in a static environment. 
Table 1. Results of alphanumeric input experiment

\begin{tabular}{|c|c|c|c|c|c|c|}
\hline & $\begin{array}{c}\text { average } \\
\text { char./min }\end{array}$ & $\begin{array}{c}\text { standard } \\
\text { error } \\
\text { char./min }\end{array}$ & $\begin{array}{c}\text { average } \\
\text { HEP }\end{array}$ & $\begin{array}{c}\text { standard } \\
\text { error } \\
\text { HEP }\end{array}$ & $\begin{array}{c}\text { average } \\
\text { SUS } \\
\text { Score }\end{array}$ & $\begin{array}{c}\text { standard } \\
\text { error SUS } \\
\text { score }\end{array}$ \\
\hline hardware keyboard & 201.1 & 11.7 & 0.020 & 0.003 & 87.3 & 3.6 \\
\hline TS keyboard & 81.3 & 4.9 & 0.039 & 0.006 & 68.5 & 4.2 \\
\hline TS handwriting rec. & 61.9 & 3.7 & 0.034 & 0.004 & 62.7 & 3.8 \\
\hline iPod touch keyboard & 72.0 & 5.6 & 0.028 & 0.009 & 66.7 & 4.7 \\
\hline turn-push-controller & 23.6 & 1.3 & 0.038 & 0.004 & 56.0 & 4.2 \\
\hline
\end{tabular}

\section{Touch vs. Knob under Regard of Interruptibility and Oscillation}

The following two experiments concentrate on a touch screen and a turn-pushcontroller under two different experimental conditions: interruptibility, tested by the occlusion method and oscillation simulated by a hexapod.

\subsection{Experimental Tasks}

For both of the following experiments, the tasks for the participants were the same. As a preliminary step, a function typology was developed from an exemplary list of functions for IVIS. This includes typical functions that appear in human-computerinteraction in the motor vehicle. From the functional typology, individual interaction types were derived systematically. For example, alpha-numeric input can be described as a sequence of the interaction types "selection from a list" or "select one from $n$ elements" and "confirmation". With this technique any kind of typical in-vehicle interaction can be described as a series of these basic interaction types.

The following interaction tasks have been used for experimental research: select one from $n$ elements (fig. 5 left), select one element from a list, two-dimensional tracking task (fig. 5 center), setting of a certain value (fig. 5 right) and confirmation by pressing the knob or touching the screen. For the selection of one element from $n$ elements the reaction time was recorded from the moment when the elements occur until the moment when the correct (or incorrect) square was pressed. In the touch screen condition, the intended element was to be touched directly, while in the knob condition a pointer switched with each step turning the knob from left to right and went down one line when the present line was crossed completely. The same time measurement was done for the selection from a list task and the setting of certain given values. For both tasks in the touch condition, a direct selection of the targeted 
elements was possible again. In the knob condition, a pointer was directed down the list of possible elements, selection was done by pressing the knob. For the setting of a given value, the turning of the knob raised the values by turning right / clockwise and decreased numbers by turning left / counter-clockwise.
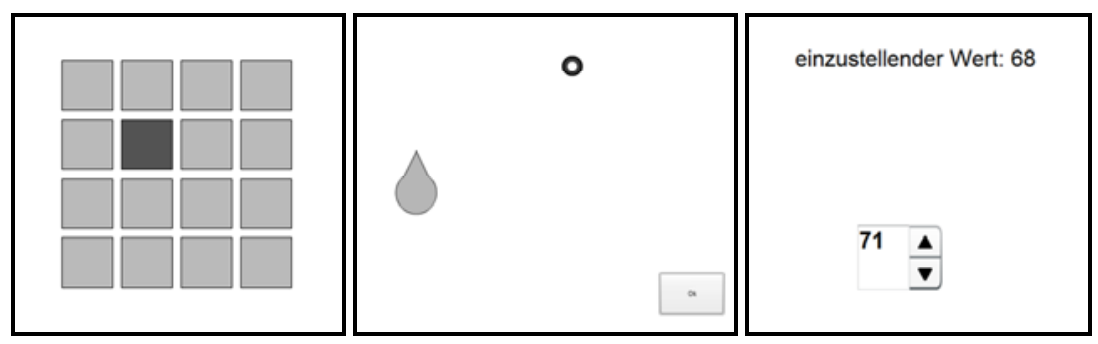

Fig. 5. Interaction prototypes: select one element from n elements, two-dimensional tracking, setting of a certain value

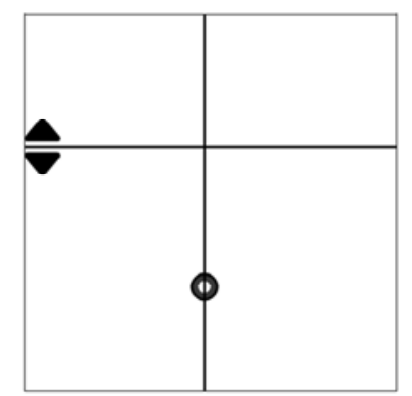

Fig. 6. Two-dimensional tracking with crosshairs in knob condition

For the two-dimensional tracking task the time needed to bring the pointer to the circle by drag \& drop (touch condition) was measured. The measurement was triggered by touching the element. With the knob the two lines of crosshairs were moved one after the other (fig. 6). The position of each line was confirmed by pressing the knob.

\subsection{Occlusion Experiment}

The second experimental setting concentrated on a comparison between touch-based interaction and turn-push-controller: here, both have been applied on a set of individual basic operations. Nowadays, these two input devices are commonly equipped in high class cars, so the usability of both is in interest.

In this experiment the above mentioned tasks have been used. They were done under regard of operating speed and error rates. In addition, the interruptibility of the tasks was measured by employment of the occlusion method. One characteristic of the following experiment is the fact that the research question aims to the automotive use of both devices, but the tests are done in a usability lab in an early stage of product development and no real car or driving simulation is needed. 
Participants. 20 participants took part in this study. The 15 male and 5 female test subjects have been university students and employees. The age was between 22 and 54 with a mean age of 29.3 years $(\mathrm{SD}=6.8)$. All participants were right-handed, two of them had red-green color blindness.

Experimental Design and Methods. After familiarizing with both input devices, all operating tasks have been conducted with and without occlusion conditions in a permuted sequence. According to ISO [7], the shutter-glasses (Plato Visual Occlusion Spectacles by Translucent Technologies Inc.) were set to occlusion and vision intervals of $1500 \mathrm{~ms}$ each. This method supports to simulate typical interruptions of the primary driving task by a secondary IVIS operating task. Nevertheless this experiment was a single-task operation. The specific metrics of this method, the total task time (TTT) and the total shutter open time (TSOT) are set into relation (R coefficient):

$$
\mathrm{R}=\mathrm{TSOT} / \mathrm{TTT}
$$

TTT is to be measured in a baseline setting without occlusion. It is the time needed for fulfillment of the whole task, while in comparison the TSOT comes from the same experiment under occlusion conditions and therefore all time with open glasses is measured. The $\mathrm{R}$ coefficient expresses the interruptibility of an operating task. If $\mathrm{R}$ is smaller than 1 , the task easily can be interrupted, while values larger than 1 characterize tasks which need much visual attention.

Beside the above mentioned objective measurements, subjective evaluation was done by usability questionnaires according to ISO 9241-11 [8].

Results. During the experiment, the single time of every operating step, the operating time for the whole tasks and the operating errors were measured. Here the participants had to do a speed-accuracy-tradeoff. The results show again that a suitable use of both evaluated devices strongly depends on the circumstances where they are applied. While being taken as a single task, the touch screen operation was faster than the usage of the knob. A further research question would be, if this result is also valid for secondary tasks. It might be possible, that the input on a knob could be more reliable, because of the interruptibility. A comparison of the occlusion-related $\mathrm{R}$ values showed, that interaction with the turn-push-controller can rather be interrupted:

$$
\mathrm{R}_{\text {turn-push-controller }}<\mathrm{R}_{\text {touchscreen }} \text {. }
$$

The distance of the touch spot to the target center point for different parameterized sections of the two-dimensional tracking task was calculated (with a bin width of 2 pixels; and an upper limit category of 'distance $\geq 36$ pixels'). The resulting figure of cumulative probability function (fig. 7) shows for example, that a target with 15 pixels radius $(6.6 \mathrm{~mm}$ diameter) will be hit right by about $60 \%$ of participants.

A closer look at the different experiments revealed, which interaction types are best fitted for the application of touch screen use in cars. We recommend to use icon menus in touch screen applications. Lists should be limited to a few entries, which fit to one screen. To enter distinct values the use of spin buttons or low resolving sliders should be favored. Two-dimensional inputs like in navigation tasks, can be easily achieved by positioning a drag \& drop marker. 


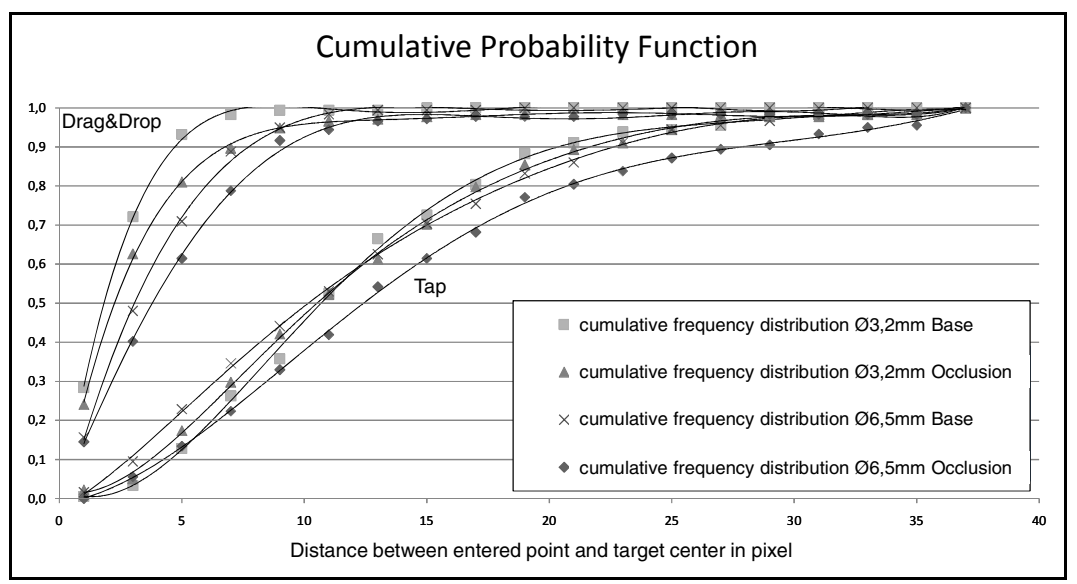

Fig. 7. Cumulative probability function

\subsection{Touch and Knob under the Influence of Oscillations}

Vibrations have an influence on human operators. Their impact can cause reduced working performance or restrain the fulfillment of tasks. A third experiment is to be introduced here as proposal for an experimental design. It focuses on the above mentioned devices, knob and touch screen, in combination with an oscillating environment. Here, both input media have been tested under vibration, which simulates a truck ride on a highway. Therefore a Steward platform (motion hexapod, six degrees of freedom, see fig. 8) was deployed with a sitting mock-up mounted on top (fig. 9). In this experiment the same tasks like in the second experiment have been conducted by the participants in a single task condition.

Participants. The 18 male participants, all from an academic environment, had an age between 25 and 64 with a mean age of 30.3 years $(S D=10.4)$. They were all right-handed and had normal or corrected to normal visual acuity. Two persons had red-green color blindness.

Experimental Design and Methods. In the last study a roadway-induced vibration was simulated to find out how this condition affects the operation with both input devices. The Steward platform was programmed with an oscillation of a truck driving on a German highway.

In the experimental procedure, the participants had to sit on a typical truck seat with fastened seat belts. The seat was mounted on the experimental mock-up. For safety reasons there was an emergency stop button mounted in a reachable distance to the participants which would stop all oscillations immediately. In no experiment this button was pressed. The movements of the hexapod were rather slight (according to a trip on a highway) so the subjects were able to concentrate on their operating tasks.

Here the participants had the following tasks: select one from $\mathrm{n}$ elements, twodimensional tracking task, select one element from a list, setting of a certain value. All tasks have been conducted with and without oscillation conditions. 


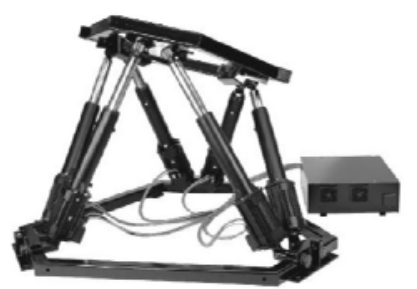

Fig. 8. Steward platform for driving oscillation

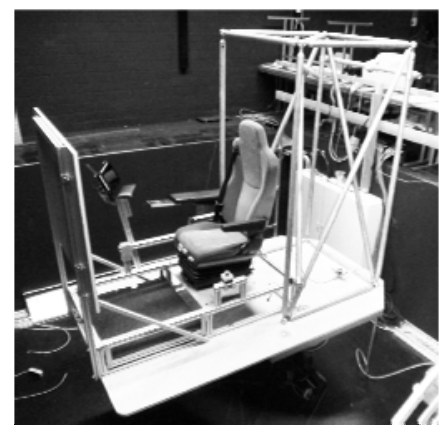

Fig. 9. Experimental mock-up

Results. With oscillation the results are very similar to the second experiment: the touch screen allows higher input speeds in the most tasks. In the list selection task with a long list (14 elements on two screens) and by setting of a certain value the knob achieves shorter input times and less input errors. The touch screen produced less input errors in the task "select one of $n$ elements" when the elements had a size according to VDI/VDE 3850 [9]. In the two-dimensional tracking task the touch screen achieved shorter input times and a lower number of errors. These results correspond with most findings of Lin et al [10]. In contrast to this experiment, their oscillation simulated maritime conditions.

\section{Conclusion and Discussion}

For alphanumeric input, a traditional keyboard showed its strength in the first mentioned study (static condition). It allows a high input speed in combination with a low error rate. If such a device cannot be taken, a touch-based handwriting recognition has a high performance potential, if the recognition itself can be improved more and the recognition process reduces its vulnerability for spidery handwriting. A larger area for the script input could also be an improvement. The other experiments also showed important criteria for the HCI towards IVIS: when operating in dual-task settings, interruptibility and environmental influences have to be mastered. By the use of the occlusion method it was shown that a turn-push-controller can support a user in a frequently interrupted task more than a touch screen can do although the latter allows faster operating processes while being taken for single tasks - a finding which was also valid under the influence of moderate oscillations. The results also shed light on necessary sizes of elements to be pressed or reached in a touch screen based operations.

The discussed experiments have shown performance data of HCI with the evaluated input devices. But they also introduced some experimental settings and methods which are well established in lab investigations in order to bring them into new application domains. The spread of innovative input devices and strategies will always bring new ideas to the automotive domain. Next developments could be multi-touch gestures. 
Yee [11] has discussed this kind of interaction and showed limitations. So further experiments could address multi-touch gestured under regard of interruptibility and oscillations in comparison to classical single-touch interaction.

Acknowledgements. The authors gratefully acknowledge the support of the TUM Graduate School at Technische Universität München, Germany.

\section{References}

1. Kavakli, M., Thorne, J.R.: A Usability Study of Input Devices on Measuring User Performance in Computer Games. School of Information Technology, Charles Sturt University, Bathurst (2002)

2. Murata, A., Iwase, H.: Usability of Touch-Panel Interfaces for Older Adults. Human Factors 47(4), 767-776 (2005)

3. Armbrüster, C., Sutter, C., Ziefle, M.: Notebook input devices put to the age test: the usability of track point and touchpad for middle-aged adults. Ergonomics 50(3), 426-445 (2007)

4. Rogers, W.A., Fisk, A.D., McLaughlin, A.C., Pak, R.: Touch a Screen or Turn a Knob: Choosing the Best Device for the Job. Human Factors: The Journal of the Human Factors and Ergonomics Society 47(2), 271-288 (2005)

5. Brooke, J.: SUS - A quick and dirty usability scale. In: Jordan, P.W., Thomas, B., Weerdemeester, B.A., McClelland, I.L. (eds.) Usability evaluation in industry, Taylor \& Francis, London (1996)

6. Hassenzahl, M., Burmester, M., Koller, F.: AttrakDiff: Ein Fragebogen zur Messung wahrgenommener hedonischer und pragmatischer Qualität. In: Szwillus, G., Ziegler, J. (eds.) Mensch \& Computer 2003: Interaktion in Bewegung, 7. bis 10, September 2003, Teubner, Stuttgart (2003)

7. ISO, 16673. Road vehicles - Ergonomic aspects of transport information and control systems - Occlusin method to assess visual distraction due to the use of in-vehicle systems (2007): ISO

8. ISO, 9241-11. Ergonomic requirements for office work with visual display terminals (VDTs) - Part 11: Guidance on usability (1999): ISO

9. VDI/VDE, 3850. User-friendly design of useware for machines. Beuth Verlag, Berlin(2000)

10. Lin, C.J., Liu, C.N., Chao, C.J., Chen, H.J.: The performance of computer input devices in a vibration environment. Ergonomics 53(4), 478-490 (2010)

11. Yee, W.: Potential Limitations of Multi-touch Gesture Vocabulary: Differentiation, Adoption, Fatigue. In: Jacko, J.A. (ed.) Proceedings of 13th International Conference on Human-Computer Interaction. HCI International 2009, Part II, San Diego, CA, USA, July 19-24, 2009, pp. 291-300. Springer, New York (2009) 